\title{
L'approche romanesque des prélèvements d'organes
}

\author{
Marie-Angèle Hermitte \\ Juriste, CNRS, UMR8103, Droit comparé de Paris (CRDST), 75004 Paris, France
}

De longue date, le roman s'est nourri des situations inédites liées au développement des sciences et des techniques. On ne compte plus le nombre d'ouvrages sur le clonage humain et ses diverses utilisations potentielles. Dans ce domaine bien exploré, le texte qui suit montre très clairement la capacité de la fiction romanesque à faire exister dans le domaine du sensible des anticipations, voire des prophéties technologiques. Le roman peut ainsi utilement compléter les réflexions sur l'éthique de la technique, par nature déconnectées l'une de l'autre. La publication de ce texte a pour objectif de montrer combien la science et la technique travaillent les imaginaires. Une autre perspective serait de mieux identifier le rôle du langage dans la création scientifique et technique - rôle dont témoignent, par exemple, l'usage des métaphores informatiques dans la biologie moléculaire, ou encore les travaux plus récents sur la contribution de la recherche à la formation de promesses technologiques, comme par exemple dans le domaine des nanotechnologies.

La Rédaction

Une revue scientifique ne publie que des articles scientifiques, de forme plus ou moins académique selon les rubriques; les livres dont la recension est faite sont eux-mêmes d'ordre scientifique, même si la vulgarisation n'est pas exclue. Or, de nombre des questions ainsi traitées $\mathrm{du}$ point de vue des sciences dites exactes ou humaines et sociales, les artistes parlent aussi, dans la littérature, bien sûr ${ }^{1}$, mais aussi par la photo, la peinture et la sculpture. Ce serait un projet en soi que de suivre régulièrement, et dans un souci d'exhaustivité, ce que l'art dit de l'impact des sciences sur les sociétés. Je ne ferai ici que proposer la lecture de deux romans récents, posant l'un et l'autre la question de l'approvisionnement de la biomédecine en matières d'origine humaine, cellules, tissus et organes. Mais, pour situer l'importance du projet, je rappellerai les expositions des cadavres "plastinés » de Gunther von Hagens $^{2}$, dont la provenance est incertaine, et le «bébé

\footnotetext{
Auteur correspondant : mahermit@gmail.com

${ }^{1}$ Et il faudrait considérer différemment la littérature générale où les sciences et les techniques sont abordées, comme dans le roman de Michel Houellebecq, Les Particules élémentaires, et les romans de science-fiction, où le centre du projet est de penser une société différente en raison des sciences et des techniques.

${ }^{2}$ La plastination est une technique de conservation créée par G. von Hagens. Voir la plaquette éditée par l'inventeur pour démontrer le caractère scientifique de son projet et inciter au don : http://www.bodymobil.de/Downloads/Franz/BD_ brochure_FRA_220807.pdf
}

mouette » de Xia $\mathrm{Yu}^{3}$, qui met en scène un cadavre de mouette à tête de fœetus humain auquel ont été greffés des yeux de lapin.

Mon premier - My Sister's Keeper, de Jodi Picoult (Atria Books, 2004) - est un roman américain réaliste sans valeur littéraire mais non sans intelligence, en raison de son habileté à traduire l'impossibilité des «solutions » aux problèmes éthiques, la plupart des décisions relevant au mieux du mécanisme du moindre mal. Traduit en français sous le titre Ma vie pour la tienne ${ }^{4}$, il cherche à confronter le lecteur à une question typique de la bioéthique, celle des «bébés-médicaments ${ }^{5}$ », qui fait référence à une technique d'analyse génétique d'embryons in vitro. Il s'agit en effet de les créer pour les trier et choisir celui qui est à la fois « en bonne santé » et «compatible» sur le plan immunitaire avec un frère ou une sœur né, atteint d'une maladie nécessitant un ou plusieurs dons. Si l'enfant malade est toujours vivant au moment de la naissance du cadet, le cordon ombilical et, dans certains cas, la moelle osseuse serviront à le traiter, avec un pourcentage non négligeable de guérisons. En l'état, on sait peu de choses du nombre

\footnotetext{
3 http://www.productionmyarts.com/arts-en-profondeur/ art-et-scandale/1999-xiao-yu-ruan-fr.htm

4 Paris, Presses de la Cité, 2004 ; J'ai lu, 2008.

5 En France, voir l'avis $n^{\circ} 72$ du Comité consultatif national d'éthique, du 4 juillet 2002 (http://www.ccne-ethique.fr/docs/fr/ avis072.pdf). L'usage de la technique a été légalisé par la loi du 8 juillet 2004 .
} 
d'embryons qu'il est nécessaire de créer en moyenne pour obtenir la naissance d'un enfant compatible; une étude américaine a compté 199 embryons testés pour 5 bébés nés. On ne sait rien non plus de la manière dont l'accouchement se passe dans ces situations d'urgence pour l'enfant malade : quel est le taux d'accouchements volontairement prématurés pour gagner du temps? Si la santé de l'enfant malade se dégrade rapidement, certains ne vont-ils pas interrompre la grossesse en cours pour tirer à temps les cellules d'un fœutus qui n'est pas encore viable $^{6}$ ? Quelle est la position de l'enfant donneur si l'aîné meurt malgré le don? Autant de questions sans réponses.

Jodi Picoult, auteur à succès, a choisi de poser le problème à travers une famille américaine bien bordée : un père pompier, une mère avocate ayant décidé de ne pas travailler pour élever les deux enfants de rigueur - pas un de plus, pas un de moins. Le monde bascule lorsque la petite Kate déclenche une leucémie du pire type qui soit : de rares et courtes rémissions si l'on dispose d'un donneur compatible, une mort rapide dans le cas inverse. Dans les heures qui suivent l'annonce de la maladie et du pronostic, Sarah renaît brutalement en mère guerrière. N'ayant pas de donneur compatible, elle expérimente la fécondation in vitro avec choix d'un embryon compatible pour pouvoir devenir donneur des cellules souches du cordon ombilical à la naissance. La maladie devient le principe de fonctionnement de la famille et, en périphérie, gravitent Anna, l'enfant qui n'aurait pas dû naître, Jesse, l'aîné subitement devenu transparent, puis alcoolique et délinquant. Que pèsent ces deux-là dont il faut sacrifier le sport, les promenades, les séances chez le dentiste, les vacances, car c'est toujours « un peu plus bas dans l'ordre des priorités », et que s'éclipsent les amis. Une vie de vigilance, $\mathrm{d}$ 'alertes et de dons pour gagner les batailles successives des rechutes et des rémissions : après le cordon ombilical, les dons de lymphocytes, de moelle osseuse et, pour finir, le rein d'Anna quand la dialyse ne suffit plus. On ne peut pas faire consentir un bébé ni une toute petite fille ; et une gamine déjà grande, une adolescente? À partir de quel âge faudra-t-il s'en préoccuper?

Le roman raconte la phase judiciaire de la révolte d'Anna la donneuse, 13 ans, car on est aux États-Unis, où tout se termine au tribunal. Lorsque sa mère organise le prélèvement d'un de ses reins, Anna va voir un avocat : "Depuis quelque temps, je fais des cauchemars dans lesquels on m'a coupée en tellement de morceaux qu'il n'est plus possible de me recomposer » (p. 22), alors mes parents, «[...] je veux les poursuivre pour atteinte à ma liberté de disposer de mon propre corps » (p. 31), ce que l'avocat reformule en « requête pour obtenir l'émancipation pour

\footnotetext{
${ }^{6}$ Pour une présentation très bien informée, mais très critique, voir le dossier réalisé par Carine Brochier et al., Le «bébémédicament » (Actualité du 19 mai 2005), Institut européen de bioéthique : http://www.ieb-eib.org/files/doc/Dossier IEB, Bébé médicament, 20050609.pdf
}

toutes décisions d'ordre médical » (p. 37). À côté de ces fermes propos, Anna multiplie les déclarations en apparence contradictoires : elle se «perd» si elle condamne sa sœur, se perd aussi si elle continue de ne pas maîtriser son destin; elle ne sait pas qui elle est à force d'être dépecée pour une autre, mais sans sa sœur elle «a du mal à se rappeler qui elle est » (p. 195). Paradoxale Anna décidée à refuser, mais incapable de le signifier à sa mère. Anna réfugiée à la caserne de son père, Anna aidée par un tuteur ad hoc mandaté par le juge pour l'aider à exprimer sa volonté véritable, Anna dont le cas n'a jamais été examiné par le comité d'éthique de l'hôpital, qui ne s'est penché que sur celui de Kate, Anna qu'il fallut immobiliser pour faire les prises de sang qu'elle refusait, Anna qui en a «marre de servir de cobaye et que personne ne lui demande comment elle supporte ça » (p. 308), Anna qui ne peut pas partir en camp de hockey car elle doit rester à portée d'hôpital au cas où sa sœur récidiverait.

Le juge hésite, tant la légitimité des deux points de vue est évidente. L'avocat égrène la litanie des interventions subies par Anna, des risques faibles mais réels qu'elle a encourus - sa mère signant pour elle les formulaires de consentement -, des risques nettement plus graves de l'ablation d'un rein. Il dessine la curieuse balance des risques - toujours pour Anna - et des avantages - pour Kate, mais aussi pour toute la famille, y compris Anna, rétorque la mère. L'avocat doit gérer le revirement du père qui, après avoir soutenu le refus d'Anna, dit finalement souhaiter le don, le refus d'Anna qui ne se sent pas la force de parler au tribunal, $l^{\prime}$ hésitation de son tuteur ad hoc qui ne sait pas mieux que les autres se déterminer, tant la place d'Anna dans la famille sera difficile lorsque Kate sera morte de son refus.

C'est l'indécision de son tuteur qui va conduire Anna à dire la vérité. Le procédé de l'auteur, qui conduit à une chute inattendue, est habile. Anna, lâchée par tous, avoue finalement que Kate, incapable de refuser elle-même la transplantation à la face de sa mère qui a tant lutté pour elle, lui a demandé de la tuer. Alors, parce qu'elle a été programmée pour donner à Kate ce dont celle-ci a besoin, elle accepte, à la demande de sa sœur, de refuser son rein, et toute l'ambivalence de son refus. «Je veux que Kate vive, mais je veux aussi être moi-même, pas une partie d'elle, je veux avoir la chance de grandir même si Kate ne le peut pas» (p. 531). Le juge ira vérifier l'histoire auprès de Kate à l'hôpital. Convaincu, il entérine le fait qu'il n'y a pas de bonne réponse à la question, cette caractéristique de la bioéthique; devant ce fait, seule compte la manière dont on prend les décisions et une règle élémentaire est qu'on ne doit pas prendre quelque chose qui appartient à un autre sans lui demander son autorisation - manière typiquement américaine de poser le problème. Dans d'autres pays, on dira qu'on ne touche pas un corps sans le consentement de la personne. Le juge émancipe donc Anna pour tout ce qui concerne sa santé, 
constatant que l'on ne peut pas demander à la mère de représenter les intérêts d'une de ses filles contre l'autre, et il confie à son avocat la charge de la représenter.

Le procès terminé, la fin sera amère. Au retour, l'avocat a un accident de voiture dans lequel Anna trouve la mort. À l'hôpital, où elle est transportée en état de mort cérébrale, l'avocat devenu son tuteur légal donne immédiatement l'autorisation de prélèvement. Kate mettra un certain temps à se remettre, mais dix ans plus tard elle est en bonne santé, la famille s'est recomposée avec les quatre personnes prévues à l'origine, le fils est redevenu le bon garçon qu'il n'aurait jamais cessé d'être sans l'interminable dérive familiale. C'est inattendu. Anna, le bébé-médicament, n'avait au fond pas de vie propre. Quand elle obtient de la justice l'autonomie de sa personne, elle perd aussitôt sa vie biologique, elle a joué son rôle. Elle n'est en rien oubliée de ceux qui l'ont aimée, mais remise à sa place, celle qui lui faisait dire qu'elle était la seule enfant à savoir non pas comment mais pourquoi elle avait été faite : «[...] je suis née parce qu'un scientifique a réussi à combiner les ovules de ma mère avec les spermatozoïdes de mon père pour produire une matière génétique de très grande valeur. [...] ils m'ont expliqué qu'ils avaient choisi spécifiquement le petit embryon de ma personne parce que je pouvais sauver ma sœur Kate [...] du coup je me suis demandé ce qui se serait passé si Kate avait été en bonne santé [...]. Si vos parents vous ont eue pour une raison précise, alors cette raison a intérêt à exister. Parce que si elle disparaît, vous disparaissez aussi » (p. 14).

Ce n'est qu'un roman. S'il fait bien comprendre la position «liée » des donneurs intrafamiliaux et, plus particulièrement, de ces bébés d'un nouveau genre sur lesquels nous n'aurons de retours que dans une trentaine d'années, il fait aussi, in fine, comprendre le malheur du malade épuisé par les soins. Le roman ne condamne rien, mais montre que, existant, les techniques ne peuvent qu'être utilisées, induisant des engrenages auxquels il est difficile d'échapper - et l'actualité lui donne une résonance étrange. En novembre 2008, en effet, les Anglais voyaient apparaître à la télévision une adolescente de 13 ans expliquant calmement, soutenue par ses parents, qu'elle ne voulait pas autoriser les médecins à lui faire une transplantation cardiaque. Souffrant d'une cardiomyopathie, conséquence des soins de la leucémie pour laquelle elle était soignée depuis l'âge de 5 ans, elle expliqua avoir subi «trop de traumatismes liés aux hôpitaux » (six opérations dans les seules deux dernières années). Hannah n'a que 13 ans et quelques mois d'espérance de vie sans cette transplantation, elle-même très risquée. A-t-elle la capacité juridique de refuser le traitement? C'est lors d'une confrontation avec les services de protection de l'enfance, qui avaient saisi les tribunaux pour l'obliger à subir la transplantation, que Hannah réussira à faire comprendre aux avocats des services sociaux ses motivations - rester à la maison dans une relative tranquillité en sachant qu'elle allait mourir - et sa compréhension des enjeux liés à sa décision. Faisant remarquer aux médecins qu'elle risquait aussi sa vie avec la transplantation et, de plus, la résurgence de son cancer du fait de la prise des immunosuppresseurs, elle leur fit prendre conscience qu'en 8 ans de traitements très lourds, elle avait eu le temps de réfléchir «au coût de la vie et au coût d'une mort digne ${ }^{7}$ ». $C^{\prime}$ est Kate, ici, qui resurgit, pas Anna.

Mon second est l'étrange roman de Kazuo Ishiguro, Never Let Me Go, traduit en français par Auprès de moi toujours ${ }^{8}$. Situé par son auteur en "Angleterre à la fin des années $90 »$ alors qu'il parut en 2005, il est donc présenté non pas comme un roman d'anticipation, mais plutôt comme le récit de quelque chose qui a déjà eu lieu. C'est une vision de l'Angleterre contemporaine qui est développée, pays où fut mise au point la fécondation in vitro ; pays où son inventeur, le Pr Edwards, recommanda tout de suite de faire pour chaque enfant un clone réserve d'organes, l'un des pays développés où la recherche biologique, sans être dérégulée, a légalisé très tôt nombre de techniques dont le potentiel de dérives est important : création d'embryons uniquement destinés à la recherche, ce qui permet d'imaginer plus facilement les chimères homme-animal, par exemple, récemment autorisées ; diagnostic préimplantatoire revendiqué aujourd'hui non seulement pour éviter des maladies graves, mais également des strabismes, ou obtenir pour des parents sourds des enfants sélectionnés en raison de leur surdité ; clonage thérapeutique qui permet de créer des embryons uniquement destinés à servir de médicaments, etc. C'est probablement ce contexte qui a été la toile de fond inspiratrice du romancier.

Les premières pages retracent les pensées d'une jeune femme de 31 ans, Kathy, qui se dit «accompagnante » depuis 11 ans. «Ils » sont satisfaits de son travail, ce qui suggère une organisation qui l'emploie, mais dont on ne saura jamais rien. On sait encore que "ses donneurs » récupèrent plus vite que prévu. Elle a un studio, une voiture, la faculté de choisir ses patients : bref, rien d'extraordinaire pour celle que l'on prend pour un médecin ou une infirmière. La grande chance de sa vie est d'avoir été éduquée à Hailsham - probablement un collège anglais chic -, et très vite le récit s'organise autour de cet âge d'or. C'est un pensionnat de la campagne anglaise, avec des juniors et des seniors, des dortoirs, un parc, du sport, des "gardiens », des professeurs, un enseignement où l'expression artistique de chaque enfant est valorisé - un banal établissement aux idées progressistes où les enfants pleurent, rient, se disputent, se réconcilient. Il y a Tommy

\footnotetext{
7 Court, M., Leclair, A., Éthique médicale, le cas Hannah Jones vu de France, Le Figaro, 12 novembre 2008.

8 Never Let Me Go, Knopf, 2005. Trad. fr. : Auprès de moi toujours, Paris, Éditions des 2 terres, 2006; Gallimard, « Folio », 2008.
} 
le colérique, Ruth la mythomane, Kathy la raisonnable, les liens qui se font et se défont. Un sujet constant de préoccupations concerne les œuvres artistiques des enfants, auxquels on demande de se montrer créatifs. Les objets qu'ils créent - peintures, sculptures, poèmes - servent lors des grandes séances annuelles «d'échanges ». Rien de plus humiliant que d'être mal placé dans cette course (et Tommy sera longtemps mis au ban de la société des enfants pour cette raison), rien de plus valorisant que de pouvoir offrir des objets de haute valeur et acquérir ces biens qui sont les seules propriétés personnelles des enfants, ce qui leur permet de constituer des collections qui font leur fierté. Et, lorsqu'une gardienne dit à Tommy qu'il n'est pas si important qu'il ne soit pas créatif, tous ses amis se rebellent contre cette idée incongrue. Parmi ces œuvres, certaines sont préemptées par un personnage épisodique, "Madame», qui vient du dehors choisir les plus belles pour sa « galerie», mais qui semble avoir peur d'eux comme on peut avoir peur des araignées. Le personnage est mal connu des enfants et la galerie qui rassemble leurs œuvres, un endroit mythique dont ils ne connaissent pas la fonction, les gardiens l'entourant de secret.

On s'étonne parfois que le propos ne garde pas trace de vacances avec les parents, et l'on se laisse aller à l'impression qu'ils sont orphelins. Le livre est émaillé d'allusions étranges à des visites médicales hebdomadaires, à l'importance toute particulière de se maintenir en bonne santé, au fait qu'ils ne pourraient pas avoir d'enfants, à des dons qui semblent mettre tout le monde dans l'embarras, au « dehors ", à un lieu antérieur à Hailsham, où les enfants n'arrivent que vers 6 ou 7 ans, on ne sait pas d'où. Mais on ne fait pas le lien entre ces allusions parsemées dans le texte. On ne comprend qu'à la page 131, lorsqu'une gardienne atypique, Miss Lucy, ne supporte pas qu'un adolescent fasse le projet d'aller mener sa carrière aux États-Unis. Elle met alors les points sur les i : «Vos vies sont tracées. Vous allez devenir des adultes, et avant de devenir vieux, avant même d'atteindre un âge moyen, vous allez commencer à donner vos organes vitaux. C'est pour cela que chacun de vous a été créé [...]. Vous avez été introduits dans ce monde dans un but précis, et votre avenir à tous, sans exception, a été déterminé à l'avance » - annonce qui lui vaudra son licenciement. Tout doucement, leur monde étrange d'adultes surgit du monde banal de leur enfance : la liberté sexuelle précoce et en principe totale entre eux, la méfiance vis-à-vis du monde du dehors où le sexe a une «tout autre signification »; les couples, stables ou non, qui se forment dans ce lieu fermé, comme feront Tommy et Ruth ; les dons, qui, de sujet de plaisanteries, deviennent un sujet «sombre et grave » qu'il est préférable d'éviter.

Grands adolescents, ils vont quitter le monde clos et protégé de Hailsham ; un petit groupe d'entre eux se retrouve à 16 ans aux Cottages, où ils doivent désormais se prendre en charge tout seuls, sans l'aide d'aucun gardien, avec d'autres du même âge, venant d'autres centres éducatifs. Ils vont tester la différence entre eux et les autres, et même commencer à sortir se promener dans le monde du dehors. C'est aux Cottages qu'ils vont réaliser que, parmi leurs semblables, la réputation d'avoir été élevé à Hailsham fait d'eux des êtres à part. C'est aux Cottages aussi que commence à les perturber leur «théorie des possibles » (p. 216) : " puisque chacun de nous avait été copié à un moment donné sur une personne normale, il devait exister pour chacun de nous, quelque part là-bas, un modèle qui vivait sa vie », et ils échafaudaient des théories, sans toutefois aller jusqu'à s'interroger sur la notion de "parents ». Ils échafaudaient leurs " avenirs de rêve ", modestes bien sûr. Ils iront jusqu'à passer une journée dans la ville voisine à chercher le « possible » de Ruth, qu'ils croient trouver jusqu'à ce que la ressemblance disparaisse pour la plus grande tristesse de Ruth. Mais pourquoi cette idée étrange qu'ils n'avaient été modelés que sur « la racaille [...] Les drogués, les prostituées, les poivrots, les clochards [...] regardez dans le caniveau » - et c'est à cette occasion que, pour la première fois, le mot «clone » est prononcé (p. 259). C'est aux Cottages, enfin, que va surgir une rumeur : ceux de Hailsham pourraient obtenir un sursis de trois ou quatre ans lorsqu'ils forment un «vrai couple». Cela donne subitement un sens à la Galerie de Madame : et si les œuvres sélectionnées « révélant l'intérieur de l'âme » étaient ce qui permettait de sélectionner les couples ayant droit au sursis ?... Ce qui signifierait que Tommy a perdu toutes ses chances, sauf à retrouver et convaincre Madame!

L'absence d'encadrement des Cottages semble provoquer un certain ennui chez les pensionnaires, qui se lancent volontairement très jeunes dans le parcours pour lequel ils ont été créés : suivre une courte formation pour devenir accompagnants de donneurs, c'est-à-dire veiller à leur récupération la meilleure et le plus rapide possible, puis devenir donneurs à leur tour.

Kathy quitte donc les Cottages pour devenir accompagnante et le restera longtemps. Elle va gérer la récupération entre les dons : le premier, peu important, le deuxième, plus lourd, le troisième, qui laisse le donneur très diminué pour arriver au quatrième, qui " termine » le donneur, s'il n'est pas mort prématurément. C'est une période passée dans le monde du dehors, mais apparemment sans autre contact qu'avec les médecins et les infirmières des centres de prélèvement. Elle accompagnera Ruth après un premier don difficile, puis Tommy; et ils renoueront sur ces bases inégales, le trio d'autrefois. Dans ce temps de l'entre-deux, Ruth dira simplement que c'est Tommy et Kathy qui auraient dû «être ensemble »; s'ils avaient formé un couple, peut-être auraient-ils pu obtenir le «sursis ». À titre de réparation, elle leur donne l'adresse de Madame. Après le troisième don de Tommy, quand Ruth est «terminée » dans de terribles douleurs dont Kathy est témoin, ils se rendent effectivement chez 
Madame, pressés par la perspective du quatrième don se profilant à l'horizon. Ils la suivent jusqu'à chez elle - semblant un couple de "grosses araignées » (p. 379). Ils racontent la rumeur du sursis et le fait que, peut-être, sa galerie a quelque chose à voir avec le sursis. Elle s'anime à ce souvenir: «[...] tous ces tableaux, ces poèmes, toutes ces œuvres de vous que j'ai réunies au cours des années [...] j'y croyais, nous y croyions tous à l'époque [...]. » Mais que peut-elle répondre à Tommy qui plaide sa cause, tendu vers son idée de sursis? «Pauvres créatures. Que vous avons-nous fait? » Alors, l'histoire de Hailsham se dévoile enfin. De bonnes âmes, des progressistes, ont voulu élever les clones non pas du pur point de vue biologique, suffisant pour donner des organes, mais d'un point de vue humain, pour leur donner au moins une jeunesse. Hailsham était l'utopie éducatrice que l'on pouvait offrir à des êtres sans parents et sans descendance, car sans autre avenir que le don. « Pourquoi avons-nous fait tout ce travail, pourquoi nous former [...] ? Si nous allons juste faire des dons [...] et puis mourir, pourquoi ces cours, ces livres et ces discussions? - Pourquoi Hailsham? ». Et pourquoi la Galerie?

« Nous avons emporté vos œuvres car nous pensions que cela révélerait votre âme, ou pour l'exprimer plus subtilement pour prouver que vous aviez une âme [...]. [...] c'était une idée courante au début (d'en douter). Ce n'est pas une notion universellement admise, même aujourd'hui [...]. [...] dans tout le pays en ce moment même, des élèves sont élevés dans des conditions déplorables [...] et les choses vont aller en s'empirant » (p. 398). Madame révèle qu'ils ont été quelques-uns à l'origine d'un mouvement étroit mais faisant beaucoup de bruit, remettant en question la manière dont les dons étaient gérés. Il fallait renverser les points de vue sur ces clones, « objets obscurs dans des éprouvettes » (p. 400). "Alors, on vous a élevés et en faisant des expositions de vos œuvres à travers le pays avec des ministres, des évêques, il y avait des promesses de fonds importants. » Il faut se remettre dans la perspective des années d'après-guerre, quand la science fit de si rapides progrès, on n'avait pas le temps de faire le point, et tout d'un coup «il y avait toutes ces possibilités qui s'offraient à nous, toutes ces manières de guérir tant de maladies auparavant incurables. C'était ce que le monde remarquait avant tout, voulait le plus et pendant longtemps, les gens ont préféré croire que ces organes surgissaient de nulle part». «[...] quand les gens ont commencé à se préoccuper des [...] élèves [...] il était déjà trop tard, il n'y avait aucun moyen d'inverser le processus. Comment demander à un monde qui en est arrivé à considérer le cancer comme guérissable, comment demander à un tel monde d'écarter cette guérison? » Le mouvement a semblé faire bouger les choses, jusqu'au scandale Morningdale, du nom d'un scientifique installé discrètement en Écosse, qui voulait offrir aux gens la possibilité d'avoir des enfants avec des caractéristiques poussées à l'extrême. Il dépassait les limites légales. Et Madame d'expliquer que les gens n'avaient pas vraiment envie d'avoir des enfants « manifestement supérieurs ${ }^{9}$ ». Ils reculaient devant ça. En une année, les sponsors se retirèrent, il fallut rentrer dans l'ombre et fermer Hailsham. L'illusion du sursis tombant, Tommy retrouve sa condition d'enfant colérique, celui qui refusait d'être créatif sur commande, celui qui aimait la trop sincère Miss Lucy, et il a un long moment de révolte, gesticulant et hurlant son désespoir. Mais, rapidement, il se referme sur sa condition de donneur attendant pour bientôt le quatrième et ultime don. Ce sera, alors, le tour de Kathy.

L'auteur ne propose aucune morale explicite. Racontée par les clones de leur seul point de vue et avec leur sensibilité propre, l'histoire décrit un monde clos, où l'extérieur est flou et les receveurs d'organes invisibles. Les clones sont totalement humains comme on peut l'imaginer, à preuve leurs amours, leurs disputes, leur créativité. Sauf que... l'on peut s'interroger à l'infini : hormis la courte crise de Tommy, aucun ne s'échappe, aucun ne conteste, aucun ne se révolte. Derrière leurs individualités touchantes, l'assignation à une destination biologique qui meut des êtres sans destin s'exprime violemment dans le verbe « terminer » qui, désignant la mort suivant le dernier prélèvement, vient les rendre à leur condition de chose.

\footnotetext{
8 On peut fortement douter de cette affirmation. Comme les parents se sont "saignés aux quatre veines " pour offrir à leurs enfants une meilleure éducation que la leur, beaucoup d'entre eux seraient sans doute prêts à leur donner une « meilleure génétique » que la leur.
} 